\section{A RESPONSE TO: "COST-EFFECTIVENESS OF ORAL SEMAGLUTIDE ADDED TO CURRENT ANTIHYPERGLYCEMIC TREATMENT FOR TYPE 2 DIABETES"}

We read with interest the JMCP April 2021 article by Guzauskas et al, "Costeffectiveness of oral semaglutide added to current antihyperglycemic treatment for type 2 diabetes." We commend the decision-analytic modeling in this study, but we have 2 major concerns that may undercut its findings. First, when applying standard practices for economic evaluations, the results from Guzauskas et al are clear-oral semaglutide is not costeffective at current US prices. Second, a much greater reduction in the price of oral semaglutide than reported is required for it to be cost-effective vs empagliflozin.

Current reference case recommendations from the Institute for Clinical and Economic Review (which provided funding for the study) include presenting the incremental cost-effectiveness ratio (ICER) for an intervention relative to its comparators. ${ }^{2}$ Because the "intervention" being studied was oral semaglutide, the results are presented as a series of pairwise comparisons to oral semaglutide, the most expensive treatment option considered $(>\$ 6,000$ per year). ${ }^{1}$ Decision makers seeking to provide efficient health care may ask, "Why compare everything to the most expensive option?"

When comparing multiple mutually exclusive treatment options, health gains with less costly treatments must be considered to determine the cost-effectiveness of more costly treatments. ICERs are calculated relative to the next least costly option, ${ }^{3}$ and decision makers must decide if they are willing to pay for additional health gains. In the United States, the willingness-to-pay threshold is likely around $\$ 100,000$ per quality-adjusted life-year (QALY) gained.. ${ }^{4}$ Applying this approach to Guzauskas et al, the ICERs are as follows: sitagliptin vs background therapy approximately $\$ 38,000$ per QALY gained, empagliflozin vs sitagliptin approximately $\$ 40,000$ per QALY gained, and oral semaglutide vs empagliflozin approximately $\$ 458,000$ per QALY gained. Empagliflozin is thus the preferred treatment, since it gains the most QALYs with an acceptable ICER. This result is borne out in the probabilistic analysis. In Supplementary Figure $4,{ }^{1}$ empagliflozin was the preferred treatment $79 \%$ of the time compared with $1 \%$ for oral semaglutide when willingness to pay was $\$ 100,000$ per QALY gained. This figure, however, is neither referenced in the manuscript nor listed in the supplementary table of contents.

Guzauskas et al also estimated that if oral semaglutide was priced at $\$ 5,562$ per year, an approximate $\$ 550$ reduction, it would be cost-effective vs empagliflozin at a willingness to pay of $\$ 100,000$ per QALY gained (Table 3). ${ }^{1}$ We believe this price to be inconceivably high. Given the 0.08 QALY gain with oral semaglutide vs empagliflozin, the total incremental cost would need to be approximately $\$ 8,000$ to produce an ICER of $\$ 100,000$ per QALY gained. Nonmedication costs comprised approximately $\$ 4,000$ of the total incremental costs for oral semaglutide vs empagliflozin (Supplementary Table 4), ${ }^{1}$ meaning that incremental medication costs must be less than $\$ 4,000$ over a lifetime. Incremental medication costs were more than $\$ 31,000$ when oral semaglutide was priced at $\$ 6,103$ per year. Furthermore, in one-way sensitivity analysis, total incremental costs were more than $\$ 26,000$ when oral semaglutide was priced at approximately $\$ 4,900$ per year (Supplementary Figure 1). ${ }^{1}$ A more realistic annual price for oral semaglutide to be cost-effective is likely approximately $\$ 2,500$.

As US health care costs continue to rise, health plans should prioritize efficient health care. The results of economic analyses should clearly state which treatment is the most cost-effective-that is, empagliflozin in Guzauskas et al.

\author{
Ciaran N Kohli-Lynch, PhD \\ Northwestern University \\ Center for Health Services \\ \& Outcomes Research \\ Chicago, IL \\ ciaran.kohli-lynch@northwestern.edu \\ Natalia Ruiz-Negrón, PharmD \\ University of Utah \\ Department of Pharmacotherapy \\ L. S. Skaggs Pharmacy Institute \\ Salt Lake City, UT \\ Amy Beal, PharmD \\ SelectHealth \\ Murray, UT \\ Brandon K Bellows, PharmD, MS \\ Columbia University \\ Department of Medicine \\ New York, NY
}

\section{REFERENCES}

1. Guzauskas GF, Rind DM, Fazioli K, Chapman RH, Pearson SD, Hansen RN. Cost-effectiveness of oral semaglutide added to current antihyperglycemic treatment for type 2 diabetes. J Manag Care Spec Pharm. 2021;27(4):455-68. doi:10.18553/jmcp.2021.27.4.455

2. Institute for Clinical and Economic Review. ICER's reference case for economic evaluations: principles and rationale. January 31, 2020. Accessed June 14, 2021. https://icer.org/wp-content/ uploads/2020/10/ICER Reference Case 013120.pdf 
3. Prosser LA, Neumann PJ, Sanders GD, Siegel JE. Reporting cost-effectiveness analyses. In: Neumann PJ, Sanders GD, Russell LB, Siegel JE, Ganiats TG, eds. Cost-Effectiveness in Health and Medicine. 2d ed. Oxford University Press; 2016.

4. Vanness DJ, Lomas J, Ahn H. A health opportunity cost threshold for cost-effectiveness analysis in the United States. Ann Intern Med. 2021;174(1):25-32. doi:10.7326/ M20-1392

\section{THE AUTHORS RESPOND}

We thank Kohli-Lynch et al for their letter (published in the JMCP August 2021 issue) and their interest in our study, ${ }_{1}^{1}$ which estimated the incremental value of the novel oral semaglutide added to existing antihyperglycemic treatment for people with type 2 diabetes mellitus at increased risk for cardiovascular disease.

Our results demonstrated incremental benefits in major adverse cardiovascular events (MACE) avoided, along with greater qualityadjusted life-years (QALYs) compared with background antihyperglycemic treatment alone. Oral semaglutide use plus background treatment resulted in better outcomes than background treatment alone or sitagliptin plus background treatment, and similar outcomes to liraglutide plus background treatment or empagliflozin plus background treatment with overlapping 95\% confidence ranges for QALYs. Oral semaglutide was estimated to be cost-effective compared with liraglutide plus background treatment and to have incremental cost-effectiveness ratios (ICERs) between $\$ 100,000$ and $\$ 150,000$ per QALY vs sitagliptin plus background treatment and background therapy alone, but was unlikely to meet these thresholds compared with empagliflozin plus background treatment.

We agree with Kohli-Lynch et al that extended dominance is an appropriate approach when evaluating the full landscape of treatment options to identify the most cost-effective option within a class of drugs and/ or for a particular indication. We also agree that empagliflozin would be the preferred add-on treatment in this analysis, since it gains comparable (though lower on average) QALYs with oral semaglutide at a lower $(-\$ 35,500)$ lifetime cost.

However, this was not the aim of our analysis. We used pairwise comparisons of oral semaglutide vs each chosen comparator instead of calculating ICERs relative to the next least costly option because our overall goal was to separately evaluate the novel agent, oral semaglutide, compared with the 4 existing comparators. We did not perform an overall cross-class evaluation so as to determine the ordering of treatment value; rather, we sought to inform decision makers as they consider the place of oral semaglutide in the current treatment landscape.

Regarding the threshold cost, we thank Kohli-Lynch et al for bringing this to our attention. The calculated oral semaglutide price needed to reach a \$100,000 willingness-to-pay per QALY threshold was due to (a) the fact that empagliflozin had lower QALYs compared with oral semaglutide in $73 \%$ of simulations and (b) the microsimulation modeling approach used to calculate each price threshold. Each threshold value was back-calculated from individual runs (15,000 total) of individual patient-level simulations per comparison. In the case of oral semaglutide vs empagliflozin, the 95\% credible range for incremental QALYs spans zero, with 27\% of the simulations having lower QALYs for oral semaglutide, whereas oral semaglutide had fully positive incremental QALY credible ranges vs the other comparators. Therefore, costeffectiveness plane quadrant crossing unfortunately makes a threshold price difficult to simply calculate from the overall costs and outcomes of the 2 treatments.

\author{
Gregory F Guzauskas MSPH, PhD \\ The Comparative Health Outcomes, \\ Policy, and Economics (CHOICE) \\ Institute \\ Department of Pharmacy, \\ University of Washington, Seattle \\ Ryan N Hansen, PharmD, PhD \\ The Comparative Health Outcomes, \\ Policy, and Economics (CHOICE) \\ Institute \\ Department of Pharmacy, \\ University of Washington, Seattle \\ rhansen@uw.edu
}

\section{REFERENCE}

1. Guzauskas GF, Rind DM, Fazioli K, Chapman RH, Pearson SD, Hansen RN. Cost-effectiveness of oral semaglutide added to current antihyperglycemic treatment for type 2 diabetes. J Manag Care Spec Pharm. 2021;27(4):455-68. 